\title{
POTENTIAL OF NEAR INFRARED SPECTROSCOPY-NIR ALLIED THE MULTIVARIATE ANALYSIS FOR GEOGRAPHICAL DISCRIMINATION OF BURITI (MAURITIA FLEXUOSA L.) AMAZONIAN OIL
}

\author{
PANERO, Pedro dos Santos ${ }^{1,5}$; PANERO, João dos Santos ${ }^{2,5}$; PANERO, Francisco dos \\ Santos $^{3,5}$; GUEDES, Rodrigo Gomes ${ }^{4}$; DE FARIA, Fernando Sérgio Escócio Drummond \\ Viana ${ }^{5}$; RODRIGUEZ, Anselmo Fortunato Ruiz ${ }^{5^{*}}$ \\ ${ }^{1}$ Instituto Federal de Ciência e Tecnologia de Roraima - Campus Boa Vista Zona Oeste, Rua Fernão Dias Paes \\ Leme, 11, cep 69303-220, Boa Vista - RR, Brasil. (fone: +55 953624 1224) \\ ${ }^{2}$ Instituto Federal de Ciência e Tecnologia de Roraima - Campus Amajari, Rodovia Antonino Menezes da Silva, \\ cep 69343-000, Amajari - RR, Brasil. (fone: +55 953593 1143) \\ ${ }^{3}$ Universidade Federal de Roraima, Departamento de Química, Av. Cap. Ene Garcez, 2413, cep 693120-000, \\ Boa Vista - RR, Brasil. (fone: +55 953621 3100) \\ ${ }^{4}$ Instituto Brasileiro de Geografia e Estatística, Departamento de Pesquisas Econômicas, Av. Getúlio Vargas, \\ 5795, cep 69301-030, Boa Vista - RR, Brasil. (fone: +55 953212 2100) \\ ${ }^{5}$ Programa de Pós-Graduação em Biodiversidade e Biotecnologia da Amazônia Legal, Rede BIONORTE, \\ Universidade Federal do Acre - UFAC, Rodovia BR 364, Km 04, Rua São Sepé, 8902, cep 69920-900, Rio \\ Branco - AC, Brasil. (fone: +55 683901 2719) \\ ${ }^{*}$ Autor correspondente \\ e-mail: ruiz@ufac.br
}

Received 20 June 2017; accepted 19 October 2017

\section{RESUMO}

Óleos da polpa do fruto de buriti (Mauritia Fleuxosa L.), oriundo da região amazônica, foram obtidos de diferentes locais do interior do estado de Roraima - Brasil, para aplicação da espectroscopia de Infravermelho Próximo com Transformada de Fourier (FTNIR) aliada a métodos quimiométricos de Análise de Componentes Principais (PCA) e Análise de Cluster Hierárquico (HCA) como alternativa metodológica para discriminação geográfica dos pontos de coleta de buriti, que tem sido foco de pesquisas relacionadas às composições de compostos bioativos e apresentado potencialidades para a extração de óleo para uso nas indústrias de combustível, farmacêutica, cosmética e alimentícia. No total, vinte amostras destes óleos foram analisadas na faixa de 700 a $2000 \mathrm{~nm}$, e processadas via aplicação de PCA e HCA. Nos dois casos as potencialidades das técnicas foram confirmadas, obtendo-se resultados satisfatórios para identificação geográfica dos óleos coletados nos municípios de Boa Vista, Mucajaí, São João da Baliza e Caroebe, com descrição de dados de até $99,77 \%$ via PCA. O que demonstra a eficácia dos métodos aplicados para discriminações sem destruição das amostras e em tempo real, útil em monitoramento eficiente de produtos saudáveis e seguros aos consumidores.

Palavras-chave: Quimiometria; Óleo Vegetal; Análise de Componente Principal; Análise de Cluster Hierárquico.

\section{ABSTRACT}

Pulp oils from buriti fruit (Mauritia Fleuxosa L.), from the Amazon region, were obtained from different locations of the state of Roraima - Brazil, for the application of Near Infrared Spectrometry with Fourier 
Transform (FTNIR) (PCA) and Hierarchical Cluster Analysis (HCA) as a methodological alternative for geographical discrimination of buriti collection points, which has been the focus of research related to bioactive compound compositions and presented potential for oil extraction for use in the fuel, pharmaceutical, cosmetic and food industries. In total, twenty samples of these oils were analyzed in the 700 to $2000 \mathrm{~nm}$ range, and processed through application PCA and HCA. In both cases the potentialities of the techniques were confirmed, obtaining satisfactory results for the geographic identification of the oils collected in the places of Boa Vista, Mucajaí, São João da Baliza and Caroebe, with a data description of up to $99.77 \%$ by PCA. Which demonstrates the effectiveness of the methods for discriminations without destruction of the samples and in real-time, useful in the efficient monitoring of healthy and safe products to consumers.

Keywords: Chemometrics; Vegetable Oil; Principal Component Analysis; Hierarchical Cluster Analysis.

\section{INTRODUÇÃO}

A autenticidade de produtos é assunto de discussão recorrente por consumidores e principalmente autoridades reguladoras de diversas áreas indústrias responsáveis pela proteção contra a fraudes, e já tem despertado interesse, em instituições internacionais, para elaboração e emissão de diretrizes visando o apoio à identificação de géneros alimentícios e produtos agrícolas pertencentes a regiões geográficas distintas, pois a qualidade e o valor comercial de alguns produtos podem estar atrelados a locais específicos (Casale \& Simonetti, 2014).

Vários métodos químicos convencionais, como técnicas cromatográficas (Berlioz et al., 2006) para determinação da proveniência regional, adulteração e autenticidade de produtos alimentícios, como azeite de oliva (Marini et al., 2006), foram empregados em décadas anteriores. Entretanto, devido a esses métodos de análises clássicas serem demorados e necessitarem de mão de obra especializada, tem-se feito esforços em busca de técnicas rápidas, precisas e capazes de manter a integridade amostral, características alcançadas pela espectroscopia no Infravermelho Próximo (NIR) aliada a análise de dados multivariados.

A utilização da espectroscopia NIR tem sido reportada em aplicações laboratoriais e industriais para classificação de materiais (Popp et al., 2012), caracterização de combustível (Kiefer, 2015), controle de qualidade de comprimidos farmacêuticos (Roggo et al., 2007; Simões et al., 2006; Trafford et al., 1999); detecção de adulteração em alimentos (Ding \& Xu, 2000; Li et al., 2016), análise e discriminação geográfica de azeite de oliva extra virgem (Casale et al., 2008; Casale \& Simonetti, 2014; Sinelli et al., 2010), etc. com sucesso e aceitação da técnica devido ao potencial matemático das análises aplicadas aos espectros das amostras. Mas ainda são escassos estudos investigando a aplicação da espectroscopia do infravermelho na região NIR para discriminação geográfica do óleo de buriti do estado de Roraima, visando a não produção de contaminantes.

O buritizeiro (Mauritia flexuosa L.), Figura 1 (a), é uma espécie de palmeira nativa da região Amazônica facilmente encontrada ao longo de rios, lagos, área de floresta ou savana em diversos estados do Brasil. É bastante conhecida pela população do estado de Roraima, devido a utilização de suas palhas na cobertura de malocas indígenas, e do seu fruto, o buriti, Figura 1 (b), fonte de consumo in natura, graças ao sabor agradável e alto valor nutricional, sendo, sua polpa, comumente encontrada à venda em feiras para manipulação do suco ou uso como ingrediente na fabricação de picolés e sorvetes.

Óleos e gorduras servem como fontes de energia concentradas e também são fontes de ácidos graxos essenciais e vitaminas, como a vitamina $E$, fundamental na dieta humana e produzida por organismos fotossintéticos (Saberi et al., 2011; Sen \& Khanna, 2006).

Pesquisas têm indicado a utilização de óleos vegetais amazônicos de copaíba, babaçu, buriti, etc., em diversos tipos de produtos industriais ou manualmente preparados, como maioneses, margarinas e molhos para saladas (Albuquerque et al., 2005; Aquino et al., 2012), por serem úteis na contribuição ao sabor, aparência, textura ou benefícios a saúde (Foster et al., 2009; Freire et al., 2016), graças à presença de componentes funcionais, capazes de agregarem elevado valor comercial aos produtos (Saberi et al., 2011). 

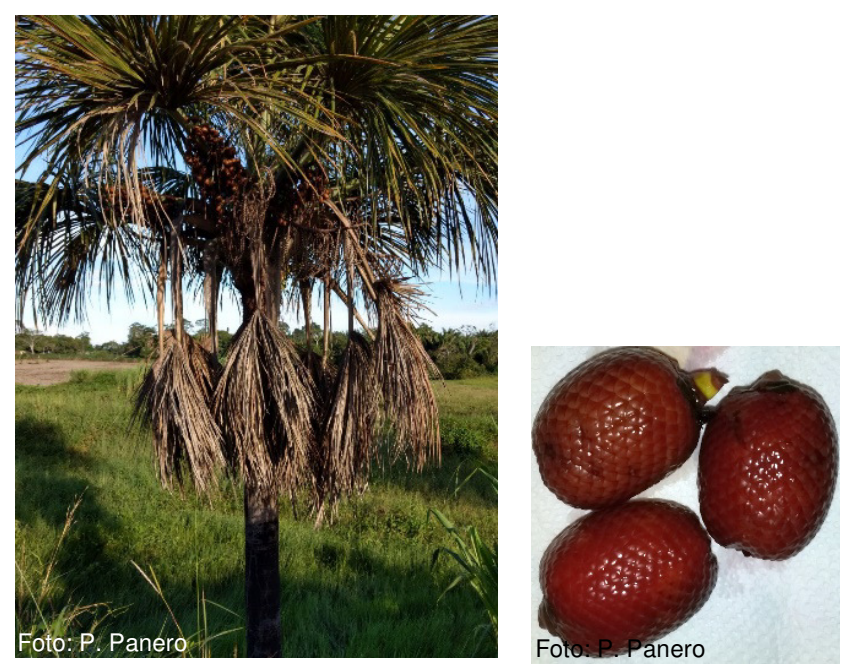

Figura 1. Palmeira (Mauritia flexuosa L.) (a), e o fruto (buriti) (b) do estado de Roraima.

O óleo de buriti apresenta propriedades funcionais devido sua concentração de ácidos graxos monoinsaturados, que são considerados importantes para redução de colesterol sanguíneo (Aquino et al., 2012; Binkoski et al., 2005), possui carotenoides, que são relacionados às funções e ações fisiológicas, como a atividade de provitamina A (De Rosso \& Mercadante, 2007) e tocoferóis, com atividade antioxidante, capaz de propiciar estabilidade oxidativa (Rodrigues et al., 2010; Silva et al., 2009), útil na indústria de alimento para preservar o óleo adequado para consumo por mais tempo (Gunstone, 2011).

O óleo de buriti também tem se mostrado profícuo para produção do biodiesel (lamaguti, 2014), pois, conforme Silva et al. (2009), em $1000 \mathrm{~kg}$ de frutos maduros é possível a extração de $45 \mathrm{~kg}$ de óleo; e utilização na indústria de cosméticos devido as suas propriedades emolientes (Zanatta, 2010) e antioxidantes, relevantes na desaceleração do fotoenvelhecimento e na proteção da pele aos efeitos nocivos da exposição solar (Natura, 2017), podendo ser aplicado como ingrediente em hidratantes corporais, sabonetes e protetores solar ou labial.

Portanto, como há potencial aplicabilidade do óleo do buriti em diversas áreas e os fatores ambientais como condições climáticas (Tura et al., 2007), origem geográfica (Galtier et al., 2008), nutrientes encontrados no solo (Cimato et al., 2003), variações na estação de coleta, manipulação pós-colheita ou processo de extração e armazenamento (Silva, et al., 2009), são capazes de interferir na qualidade de óleos vegetais - podendo vir a comprometer suas utilizações em alimentos, cosméticos ou medicamentos - é efetivamente importante a aplicação de metodologias que possibilitem rapidez no processo de identificação da procedência destes óleos.

Este trabalho aborda a espectroscopia infravermelha na região NIR aliada as análises quimiométricas dos dados espectrais do infravermelho para verificação da procedência geográfica do óleo da polpa de buriti das localidades de Boa Vista, Mucajaí, São João da Baliza e Caroebe, pertencentes ao estado de Roraima, com o intuito de demonstrar o emprego de uma técnica alternativa, rápida, sem produção de resíduos e com capacidade de manutenção da integridade amostral.

\section{MATERIAL E MÉTODOS}

\subsection{Material}

\subsubsection{Amostras de Óleo}

Foram analisadas um total de 20 amostras de óleo da polpa de buriti, de quatro localidades do estado de Roraima:

São João da Baliza - vicinal 16, localizada a $330 \mathrm{~km}$ da cidade de Boa Vista e pertencente a área de vegetação de Floresta Ombrófila Densa Submontana com Dossel Emergente com Pastagem (IBGE, 2005);

Caroebe - vicinal 6 da região de Entre Rios, localizada a $394 \mathrm{~km}$ da cidade de Boa Vista e com classificação florestal idêntica a São João da Baliza, conforme IBGE, 2005;

Boa Vista - região do PA Nova Amazônia - Gleba Truaru, área rural, localizada a $82 \mathrm{~km}$ da cidade de Boa Vista, capital do estado de Roraima, com vegetação de Savana Parque com Floresta de Galeria (IBGE, 2005);

Mucajaí - vicinal 17 da vila do Apiaú, localizada a $136 \mathrm{~km}$ da cidade de Boa Vista, na área de vegetação de Floresta Ombrófila Aberta Submontana com Palmeiras.

\subsubsection{Preparação das Amostras}

As amostras foram descascadas, despolpadas, trituradas e colocadas para secar 
por $24 \mathrm{~h}$ sob temperatura controlada de $65{ }^{\circ} \mathrm{C}$. Posteriormente, cada unidade amostral foi embalada em papel-filtro, posta no interior do extrator Soxhlet - com balão abastecido com 250 $\mathrm{mL}$ de éter etílico - e submetida ao sistema de refluxo contínuo, por $6 \mathrm{~h}$, para extração do óleo. Por fim, as amostras foram rotaevaporadas e armazenadas em frascos específicos.

\subsection{Métodos}

\subsubsection{Medidas por Infravermelho Próximo (NIR)}

A espectroscopia NIR foi utilizada para obtenção dos espectros de amostras dos óleos da polpa de buriti, na faixa de 700 a $2000 \mathrm{~nm}$, com espectro médio obtido de 30 leituras à temperatura ambiente de $28 \stackrel{\circ}{\circ}$. As respostas espectrais foram gravadas no modo absorbância e geradas pelo espectrofotômetro modelo Lambda 950, da marca PerkinElmer, com acessório de esfera de integração e detector InGaAs/PMT.

\subsubsection{Análise de Componente Principal - PCA}

Foi utilizado o PCA, método de reconhecimento de padrão não supervisionado, visando a detecção do comportamento amostral e a compressão de dados de múltiplas variáveis correlacionáveis, com propriedades úteis na descrição das informações presentes nos dados originais, visualizadas mediante auxílio de programa computacional específico que decompõe a matriz X na matriz de "scores" $\mathbf{T}$ e "loadings" L, capazes de fornecer informações de correlações e padrão de similaridade amostral. Com "scores" representando as relações entre as amostras e "loadings" as relações entre as variáveis (Ferreira, 2015; Souza \& Poppi, 2012).

$$
\mathbf{X}=\mathbf{T L}^{\mathbf{T}}
$$

\subsubsection{Análise de Cluster Hierárquico - HCA}

Também foram aplicados, aos espectros NIR, o método HCA, que fornece representações gráficas bidimensionais, com auxílio computacional, e possibilita o relacionamento do grau de similaridade amostral com 0 comprimento das ramificações apresentadas via dendrograma (PirouetteTM, 2003). O dendrograma obtido, neste caso, foi mediante cálculo de distância euclidiana entre grupos amostrais com critério de agrupamento de variância mínima incremental (Eq. 2), transformações de correção de linha base (quadratic fit) e log 10 aplicados aos dados da matriz (20 x 1301) em um programa específico, com técnicas quimiométricas,

$d_{A B \rightarrow C}=\left(\frac{\left(n_{A}+n_{C}\right) \mathrm{d}_{A C}^{\mathrm{D}}+\left(n_{B}+n_{C}\right) \mathrm{d}_{\mathrm{B} C}^{\mathrm{N}}-n_{C} \mathrm{~d}_{\mathrm{AE}}^{\mathrm{N}}}{n_{A}+n_{B}+n_{C}}\right)^{1 / 2}$

sendo " $n_{i}$ " o número de amostras em "i" agrupamentos, e os " $x_{a j}$ " e " $x_{b j}$ ", respectivamente, os valores da j-ésima coordenada de A e B.

\section{RESULTADOS E DISCUSSÃO:}

\subsection{Espectrocopia NIR}

Na Figura 3 estão dispostos os espectros de infravermelho próximo, na faixa de 700 a $2000 \mathrm{~nm}$, de quatro amostras de óleos da polpa de buriti, de diferentes regiões do estado de Roraima (São João da Baliza, Caroebe, Boa Vista e Mucajaí), dos quais pode-se verificar comprimentos de ondas associados aos modos vibracionais, em concordância com Albuquerque et al. (2005), que relataram a mesma assinatura espectral do óleo vegetal de buriti em seu trabalho e consideraram a banda entre 920 e $940 \mathrm{~nm}$ como característica de compostos que possuem alfa-tocoferol e ácido oleico e, o pico presente em $1210 \mathrm{~nm}$, característico de compostos que apresentam alta concentração de ácido oleico (Albuquerque et al., 2005).

Os picos presentes entre 920 a $1215 \mathrm{~nm}$ são oriundos dos segundos overtones da banda $\mathrm{C}-\mathrm{H}$, característico de lipídios, conforme Wilson et al. (2015). Em 1390 e 1412 nm estão evidenciados os picos usualmente atribuídos à compostos que possuem alta concentração de ácido oleico (Albuquerque et al., 2005). O pico $1430 \mathrm{~nm}$ pode ser atribuído ao primeiro overtone do estiramento $\mathrm{O}-\mathrm{H}$. E os picos em 1722 e 1760 nm são característicos de compostos que apresentam ácido oleico, associados ao primeiro overtone de estiramento $\mathrm{C}-\mathrm{H}$ e/ou $\mathrm{O}-\mathrm{H}$ (Albuquerque et al., 2005; Wilson et al., 2015).

\subsection{Análise de Componentes Principais de Dados NIR}

$\mathrm{Na}$ Tabela 1, verifica-se a descrição de $100 \%$ da variação total dos dados fornecida pelas componentes principais PC1, PC2 e PC3 
que possibilitaram a discriminação geográfica das amostras, mediante a visualização das informações mais relevantes oriundas da variabilidade da matriz de dados (20 x 1301), construídas com a totalidade de 20 amostras e 1301 variáveis em quintuplicatas.

Tabela 1. Descrição da variabilidade dos dados (\%) via associação de componentes principais 1 , 2 e 3.

\begin{tabular}{c|c}
\hline $\begin{array}{c}\text { Componentes } \\
\text { Principais }\end{array}$ & $\begin{array}{c}\text { Descrição da } \\
\text { variabilidade } \\
\text { dos dados (\%) }\end{array}$ \\
\hline PC1 + PC2 + PC3 & $100,00 \%$ \\
PC1 + PC2 & $99,77 \%$ \\
PC1 & $95,29 \%$ \\
\hline
\end{tabular}

A primeira componente principal (PC1) em associação com a segunda componente principal (PC2), Tabela 2, foram responsáveis por descreverem $99,77 \%$ da variação total, com 95,29\% atribuídos à $\mathrm{PC} 1$ e apenas $4,48 \%$ à PC2.

Na Figura 4 (PC2xPC1) está representado o gráfico de scores com a discriminação de dois grandes grupos evidenciados pela análise da PC1: o da região Sul do estado de Roraima, caracterizada por possuir fitoecologia de Floresta Ombrófila Densa Submontana com Dossel Emergente com Pastagem, onde estão contidos os agrupamentos de São João da Baliza e Caroebe; e o da região Centro-Norte do estado de Roraima, no qual estão inseridas as amostras de Boa Vista e Mucajaí, pertencentes, respectivamente, às áreas de vegetação de Savana Parque com Floresta de Galeria e de Floresta Ombrófila Aberta Submontana com Palmeiras.

Ainda na Figura 4, é possível notar, analisando também a PC2, as discriminações dos locais de onde foram realizadas as coletas, provavelmente devido aos fatores climáticosregionais e variações dos nutrientes presentes no solo, onde observa-se o agrupamento das amostras de Boa Vista e Mucajaí, com pontos de coletas distantes por mais de $100 \mathrm{~km}(218 \mathrm{~km}$ por estrada); e das amostras de São João da Baliza e Caroebe, com distância entre os pontos de coleta de, aproximadamente, $56 \mathrm{~km}$.

\subsection{Análise de Agrupamento Hierárquico de Dados NIR}

A Figura 5 apresenta o dendrograma obtido por HCA, com transformação de correção de linha base (quadratic fit) e log 10. Nesta, verifica-se a confirmação das tendências obtidas via PCA, pois, pode-se observar agrupamentos que evidenciam a discriminação geográfica das amostras das localidades de Boa Vista e Mucajaí, pertencentes à região Centro-Norte do estado de Roraima, e de Caroebe e São João da Baliza, oriundas da região Sul de Roraima.

\section{CONCLUSÕES:}

A aplicação da técnica PCA-NIR apresentou $99,77 \%$ da descrição da variabilidade dos dados e foi confirmada pela técnica HCANIR. O que demonstra que a espectroscopia na região do infravermelho próximo (NIR), quando aliada à análise de componentes principais (PCA) ou de agrupamento hierárquico (HCA) fornecem informações rápidas e eficientes quanto a discriminação geográfica de óleos da polpa de buriti coletados nas localidades de Boa Vista, Mucajaí, São João da Baliza e Caroebe, do estado de Roraima. Os resultados indicam que esse tipo de aplicação tem grande potencial para rastreabilidade e certificação da procedência geográfica das amostras de óleos vegetais, mediante o controle de qualidade confiável, rápido e sem utilização de produtos nocivos ao ambiente e ao operador.

\section{AGRADECIMENTOS:}

Ao programa de Pós-Graduação em Biodiversidade e Biotecnologia da Amazônia Legal da Universidade Federal do Acre - UFAC, pela liberação do laboratório e equipamentos que contribuíram fundamentalmente para viabilidade do trabalho. Ao Instituto Federal de Ciência e Tecnologia de Roraima - Campus Boa Vista Zona Oeste pelo apoio à capacitação, concedida ao primeiro autor.

\section{REFERÊNCIAS:}

1. Albuquerque, M.L.S., Guedes, I., Alcantara, Jr., Moreira, S.G.C., Neto, N.M.B., Correa, D.S.; Zilio, S.C. Journal of the Brazilian Chemical Society, 2005,16, 6a, 1113-1117.

2. Aquino, J.S., Pessoa, D.C.N.P., Araújo, K.L.G.V.; Epaminondas, P.S., Schuler, A.R.P., De Souza, A.G., Stamford, T.L.M. Journal of the Brazilian Chemical Society, 
2012, 23, 2, 212-219.

3. Berlioz, B., Cordella, C., Cavalli, J.F., Lizzani-Cuvelier, L., Loiseau, A.M., Fernandez, X. Journal Agricultural and Food Chemistry, 2006, 54, 10092-10101.

4. Binkoski, A.E., Kris-Etherton, P.M., Wilson, T.A., Mountain, M.L., Nicolosi, R.J. Journal of The American Dietetic Association, 2005, 105, 7, 1080-1086.

5. Casale, M., Casolino, C., Ferrari, G., Forina, M. Journal of Near Infrared Spectroscopy, 2008, 16, 39-47.

6. Casale, M. \& Simonetti, R. Journal of Near Infrared Spectroscopy, 2014, 22, 5980.

7. Cimato, A., Franchini, E., Lapucci, C., Attilio, C., Sani, G., Costantini, E. A.C., Pellegrini, S., Barbetti, R. Proceedings of the National Conference on "Qualità del suolo, impatto antropico e qualità dei prodotti agricoli", 2003, 428-433.

8. De Rosso, V.V. \& Mercadante, A.Z. Journal Agricultural and Food Chemistry, 2007, 55, 5062-5072.

9. Ding, H.B. \& Xu, R.J. Journal of Agricultural and Food Chemistry, 2000, 48, 2193-2198.

10. Ferreira, M.M.C. Quimiometria Conceitos, Métodos e Aplicações. Editora da UNICAMP, Campinas, SP, 2015.

11. Foster, R., Williamson, C. S., Lunn, J. Nutrition Bulletin. 2009, 34, 4-47.

12. Freire, J.A.P., Barros, K.B.N.T., Lima, L.K.F., Martins, J.M., Araújo, Y.C., Oliveira, G.L.S., Aquino, J.S., Ferreira, P.M.P. Journal of Food Science, 2016, 81, 11, R2611-R2622.

13. Galtier, O., Le Dréau, Y., Olliver, D., Kister, J., Artaud, J., Dupuy, N. Applied Spectroscopy, 2008, 62, 583-590.

14. Gunstone, F.D. Vegetable Oils in Food Technology: Composition, Properties and Uses, 2a. ed, Wiley-Blackwell: Oxford, 2011.

15. lamaguti, P. S. Dissertação de mestrado. Universidade Estadual Paulista, 2014.

16. IBGE, Instituto Brasileiro de Geografia Estatística. Estado de Roraima Vegetação. Ministério do Planejamento, Orçamento e Gestão, 2005, 1.

17. Kiefer, J. Energies, 2015, 8, 3165-3197.

18. Li, X., Feng, F., Gao, R., Wang, L., Qian, Y., Li, C., Zhou, G. Journal of the Science of Food and Agriculture, 2016, 96, 9, 3148-3156.
19. Marini, F., Magrì, A.L.; Bucci, R., Balestrieri, F., Marini, D. Chemometrics and Intelligent Laboratory Systems, 2006, 80, 1, 140-149.

20. Marsaro Júnior, A.L., Peronti, A.L.B.G., Penteado-Dias, A.M., Morais, E.G.F., Pereira, P.R.V.S. Brazilian Journal of Biology, 2013, 73, 2, 413-418.

21. Natura. http://www.natura.com.br/e/buritisaude-e-beleza-no-cerrado. Acessado em junho 2017.

22. PirouetteTM. Multivariate Data Analysis. Pirouette User Guide, Version 3.11. Infometrix Inc, Woodinville, WA, 2003.

23. Popp, J., Tuchin, V., Chiou, A., Heinemann, S. Em Handbook of Biophotonics. WILEY-VCH. Germany: Weinheim, 2012, vol 3.

24. Rodrigues, A.M.C., Darnet, S., Silva, L.H.M. Journal of the Brazilian Chemical Society, 2010, 21, 10, 2000-2004.

25. Roggo, Y., Chalus, P., Maurer, L., LemaMartinez, C., Edmond, A., Jent, N. Journal of Pharmaceutical and Biomedical Analysis, 2007, 44, 683-700.

26. Saberi, A.H., Kee, B.B., Oi-Ming, L., Miskandar, M.S. Food Chemistry, 2011, 127, 1031-1038.

27. Sen, C.K. \& Khanna, S., Roy, S. Life Sciences, 2006, 78, 2088-2098.

28. Silva, S., Sampaio, K.A., Tahan, T., Rocco, S. A., Ceriani, R., Meirelles, A.J.A. Journal of the American Oil Chemists' Society, 2009, 86, 611-616.

29. Simões. S.S., Sanches, F.A.C., De araújo, M.C.U., Pasquini, C., Junior, I.M.R., Rohwedder, J.J.R. Resumos da 29a Reunião Anual da Sociedade Brasileira de Química, Águas de Lindóia., Brasil, 2006.

30. Sinelli, N., Casale, M., Di Egidio, V., Oliveri, P., Bassi, D., Tura, D., Casiraghi, E. Food Research International, 2010, 43, 2126-2131.

31. Souza, A.M. \& Poppi, R.J. Química Nova, 2012, 35, 1, 223-229.

32. Trafford, A.D., Jee, R.D., Moffat, A.C., Graham, P. The Analyst, 1999, 124, 163-167.

33. Tura, D., Gigliotti, C., Pedò, S., Failla, O., Bassi, D., Seraiocco, A. Scientia Horticulturae, 2007, 112, 108-119.

34. Wilson, R.H., Nadeau, K.P., Jaworski, F.B., Tromberg, B.J., Durkin, A.J. Journal of Biomedical Optics, 2015, 20, 3, 1-10.

35. Zanatta, C.F., Mitjans, M., Urgatondo, V., 
Rocha-Filho, P.A., Vinardell, M.P. Food and Chemical Toxicology, 2010, 48, 1, 7075.

PERIÓDICO TCHÊ QUÍMICA • www.periodico.tchequimica.com • Vol. 15 N. 29.

• ISSN 1806-0374 (impresso) • ISSN 1806-9827 (CD-ROM) • ISSN 2179-0302 (meio eletrônico) 


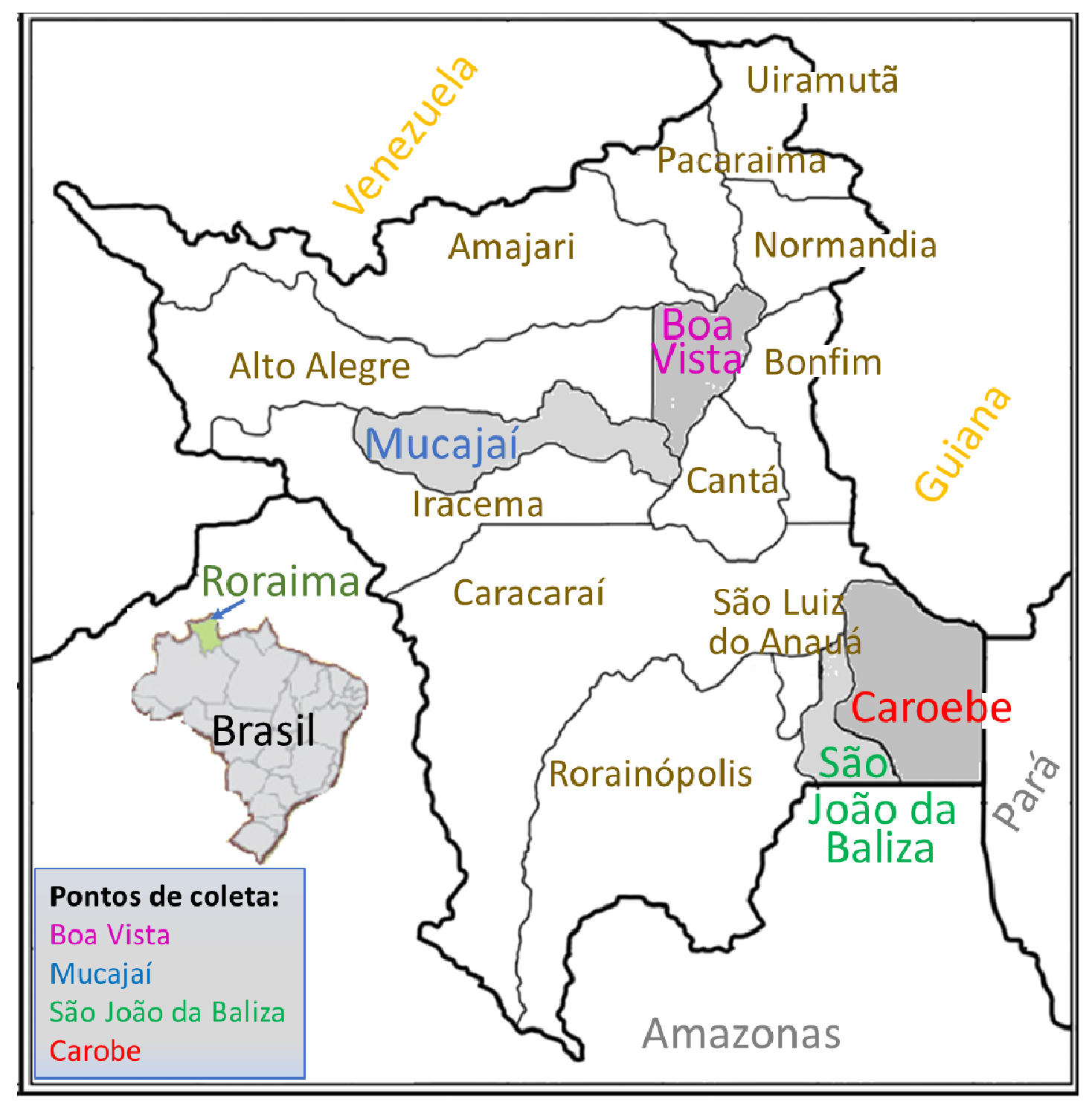

Fonte: Marsaro Júnior et al., 2013.

(Adaptada pelos autores)

Figura 2. Mapa do estado de Roraima com representações das regiões de coleta das amostras de buriti. 

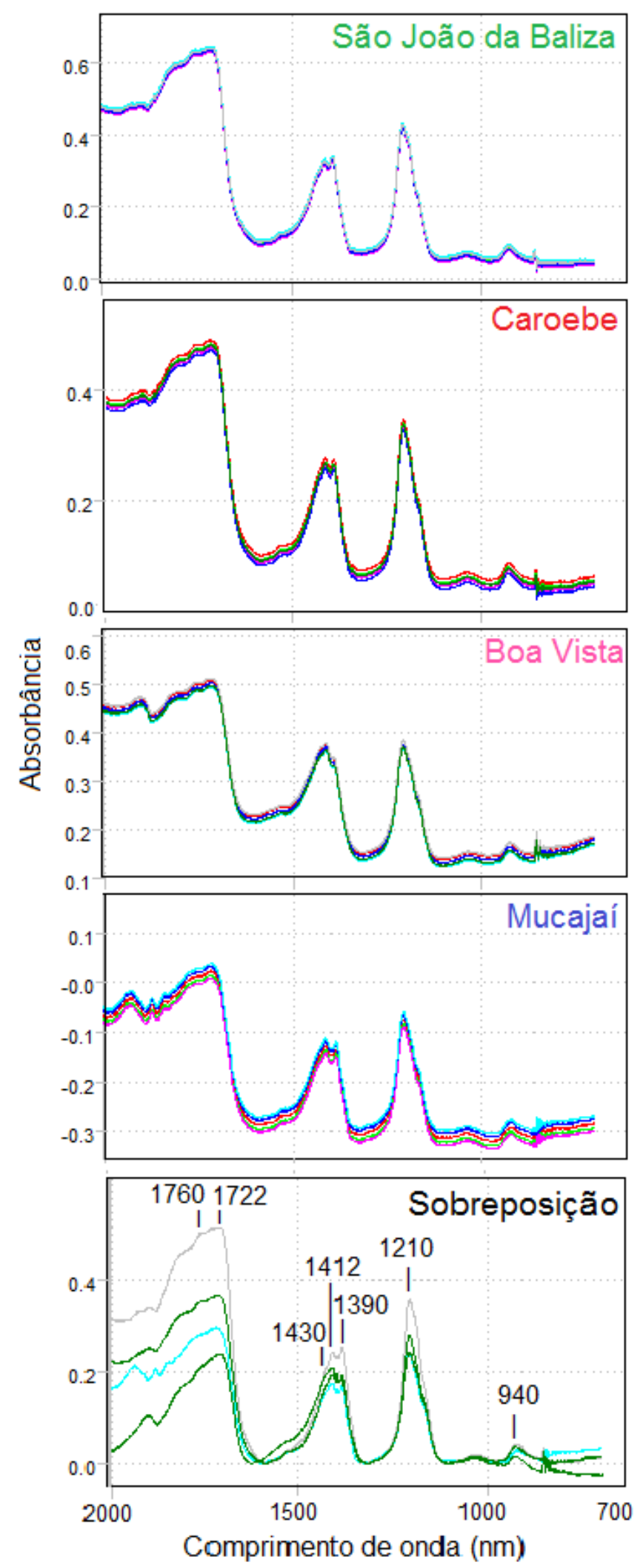

Figura 3. Espectros de amostras de óleos de buriti de São João da Baliza, Caroebe, Boa Vista, Mucajaí e a sobreposição dos espectros locacionais com algumas bandas evidenciadas. 


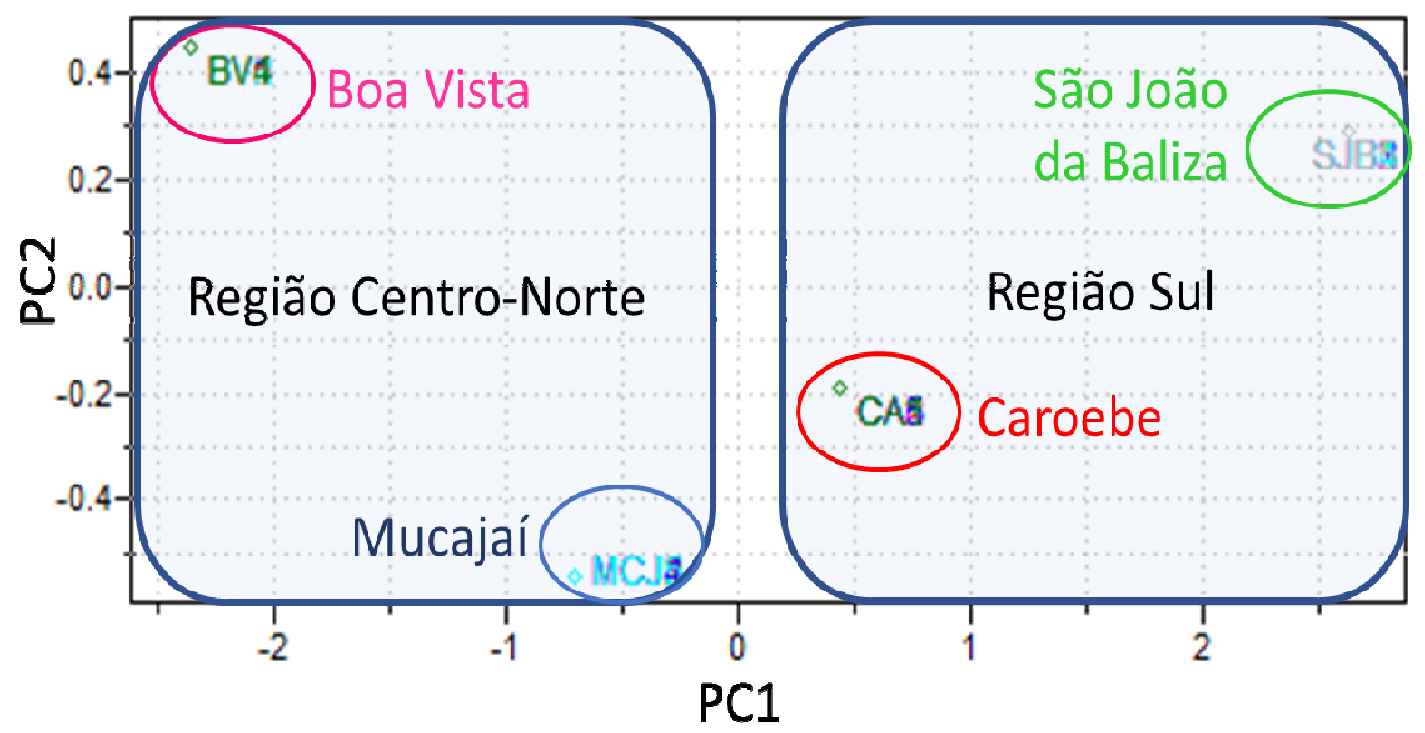

Figura 4. Gráfico dos scores, $P C 2$ vs $P C 1$, centrado na média, com correção da linha base (quadratic fit) e log 10, na banda de 700 - $2000 \mathrm{~nm}$, com descrição de variabilidade de dados de 99,77\% cumulativa de PC1 e PC2, evidenciando a discriminação geográfica dos óleos de buriti presentes no estado de Roraima, por localidades e regiões.

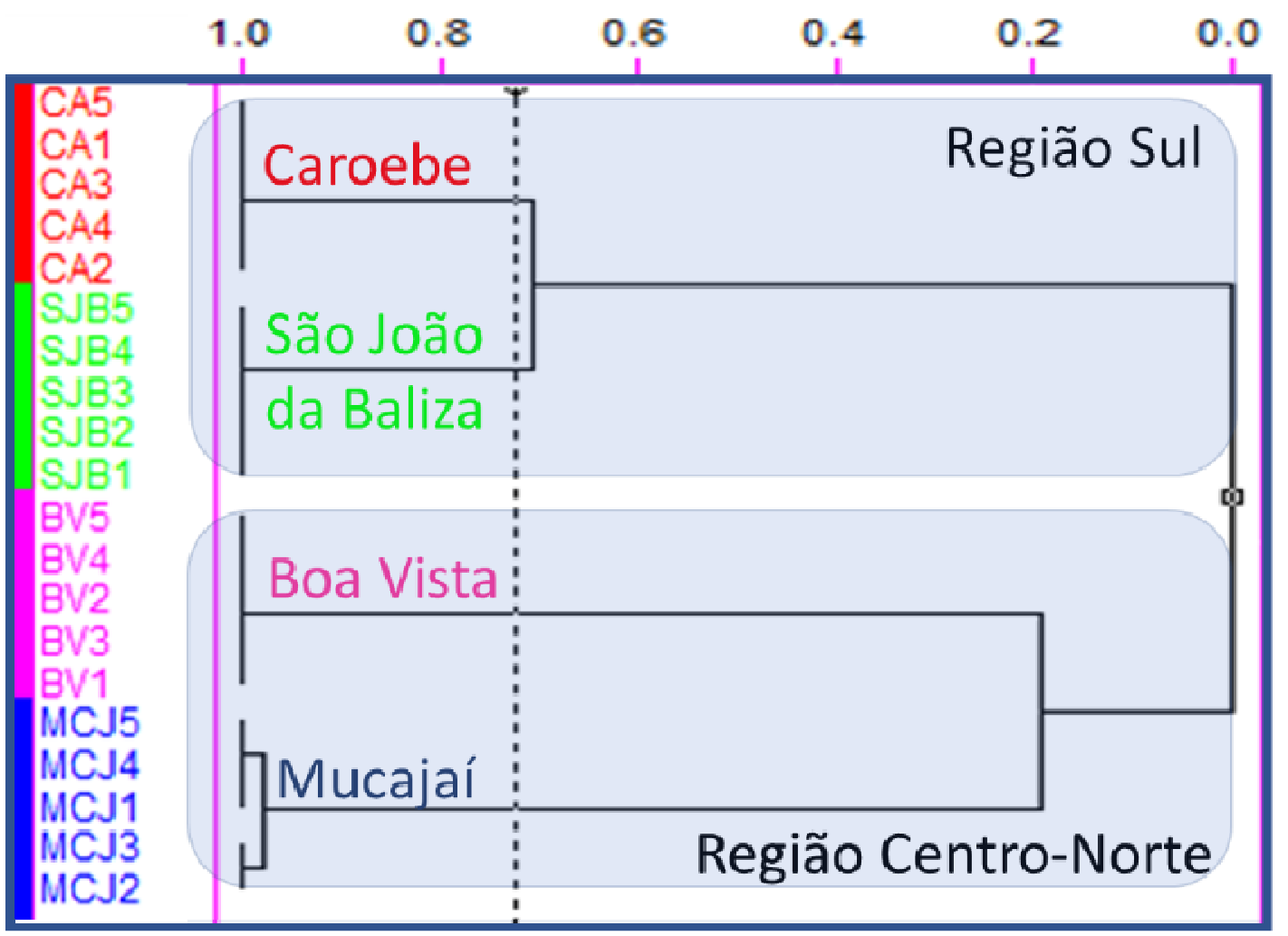

Figura 5. Dendrograma das amostras de óleos de buriti, com centragem na média, distância métrica euclidiana, técnica de conexão incremental, transformações de correção de linha base (quadratic fit) e $\log 10$, evidenciando a discriminação geográfica dos buritis provenientes do estado de Roraima e localidades específicas. 